\title{
Construction Robot Systems Using Mobile Manipulators - Piling Up Blocks with Real Systems and Positioning Accuracy -
}

\author{
Masatoshi Hatano*, Mamoru Minami**, Toshiyuki Asakura** \\ and Tsuyoshi Ohsumi*
}

\author{
* Department of Mechanical and Intellectual Systems Engineering, \\ Faculty of Engineering, Toyama University \\ $\$ 190$ Gofuku, Toyama, 930-8555, Japan \\ E-mail : masatosi@eng.toyama-u.ac.jp \\ ** Department of Mechanical Engineering, Faculty of Engineering, Fukui Univcrsily \\ 3-9-1 Bunkyo, Fukui, 910-8507, Japan \\ E-mail : minami@scmc.mech.fukui-u.ac.jp
}

\begin{abstract}
This research is concerned with a construction robot system that uses a mobile manipulator in order to make a large fence made up of concrete blocks. One of problems in making such a fence by a mobile manipulator is the cxistence of stacking errors caused by traveling errors under nonholonomic constraints. In the present paper, we propose a basic construction robot system that uses a mobile manipulator equipped with a systern of compensating for stacking errors by detecting traveling errors using landmarks and a hand-eye camera. In addition, the system's accuracy in positioning the blocks is evaluated through experiments with a prototype of experimental equipment.

Keywords: Mobile Manipulator, Construction Robot System, Block, Hand-eye Camera, Positioning Accuracy, Landmark
\end{abstract}

\section{Introduction}

Mobile manipulators [1]-[5] are able to propel themselves around a work area and, unlike floorfixed manipulators, are able to operate over a wide area. The construction of a large fence made up of stacked concrete blocks requires hard labor and a wide work area. Mobile manipulators are effective in constructing such large concrete-block fences, because the mobile manipulator's working spaces are not limited by their traveling mechanism. At present, a robot capable of stacking stones and which has a crawler mechanism, has been realized. However, it is not autonomous, but must be operated by human beings.

The purpose of this research is to construct a construction robot system with a mobile manipulator. The basic operations for a mobile manipulator are classified roughly as follows: (1) a carrying operation for picking up and loading objects on to its own body; (2) a transporting operation for conveying the loaded objects; and (3) a stacking operation for unloading and positioning the objects after transportation. In the present paper, the basic operations (1) through (3) are referred to as the basic operation of stacking blocks. The most important problem in the performance of this basic operation is the final positioning accuracy of objects after the completion of operations (1) through (3). On the other hand, it is known generally that navigating a mobile manipulator to a destination accurately in the operation (2) is difficult, because the mobile manipulator travels under nonholonomic constraints [6]. Thus, accurate travel can not be realized in a real system $[4],[7],[8]$. It was shown in a previous study [4] that positioning errors of a mobile manipulator's hand caused by traveling errors on a horizontal plane can be decreased by the use of a mounted manipulator with a hand-eye visual feedback control system. However, we did not examine the positioning accuracy of objects after the completion of operations (1) through (3) in that study [4].

In the present paper, a compensation method for stacking errors caused by traveling errors is proposed by using landmarks and a hand-eye visual feedback systern. Here, fixation motion is utilized in order to reduce the aftects of deformation of the hand-eye camera's lens and to recognize traveling errors accurately.

In addition, we evaluate the positioning accuracy of the stacked blocks after the completion of operations (1) through (3) by a prototype of our experimental equipment. Then, we discuss the relationships between the stacked positions and changes in posture of the mobile manipulator. The changes are caused by (a) dynamical interactions between the mounted manipulator and the mobile robot, and (b) changes in the position of the center of gravity, which depend on changes in the posture of the mounted manipulator, during operation.

\section{Kinematics}

\subsection{Definition of Kinematic Relationships}

Fig. 1 shows the relationships between the mobile manipulator, a landmark, the desired position for a stack block. In Fig. $1, \Sigma_{W}$ is a frame of reference, $\boldsymbol{\Sigma}_{M}$ is a frame of the mobile manipulator, $\boldsymbol{\Sigma}_{6}$ is a frame of the hand, $\boldsymbol{\Sigma}_{C}$ is a frame of the video camera, $\boldsymbol{\Sigma}_{P 0}$ is a frame of the landmark and $\boldsymbol{\Sigma}_{B 0}$ is a frame of the desired position for a stack of blocks. Here, $\boldsymbol{\Sigma}_{M}, \boldsymbol{\Sigma}_{6}$ and $\boldsymbol{\Sigma}_{C}$ are moving frames, which move with the mobile manipulator, and $\boldsymbol{\Sigma}_{W}, \boldsymbol{\Sigma}_{P 0}$ and $\boldsymbol{\Sigma}_{B 0}$ are 


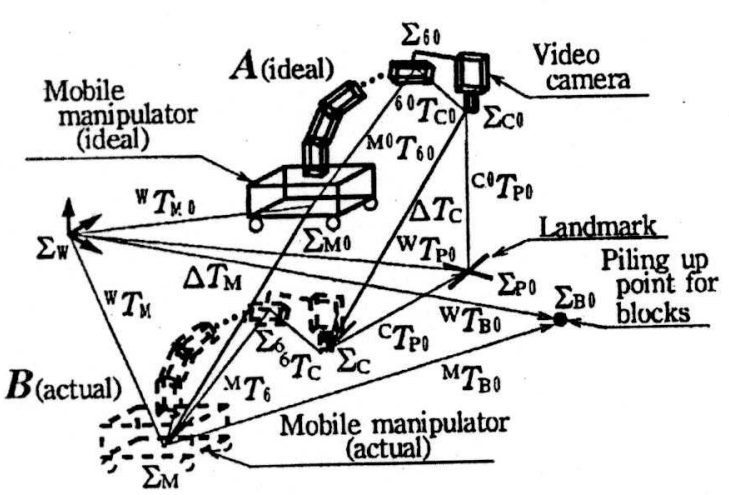

Fig. 1 Kinematics of mobile manipulator system

frames that are fixed to the road. In addition, ${ }^{i} T_{j}$ $(i=W, M, 6, C: j=M, 6, C, P 0, B 0)$ is a homogeneous translation matrix, which expresses the position/orientation of $\boldsymbol{\Sigma}_{j}$ with reference to $\boldsymbol{\Sigma}_{i}$, and it is used in the following formulation of kinematics. Especially, ${ }^{W} T_{P 0}$ is the position of the landmark and ${ }^{W} \boldsymbol{T}_{B 0}$ is the desired position for a stack of blocks. ${ }^{W} T_{B}$ shows the actual position $\Sigma_{B}$ of a stack of blocks with reference to $\Sigma_{W}$, but it is not shown in Fig. 1.

The relationship between the hand, the camera and the posture of the mounted manipulator is referred to as the reference posture of the manipulator when the mobile manipulator travels to a desired position without the occurrence of traveling errors and the mounted manipulator realizes the desired posture. The relationship between the mobile manipulator and the landmark in the reference posture is referred to as the reference relationship of position/orientation and it is shown as $A$ in Fig. 1. The relationship is described as:

$$
{ }^{{ }^{W}} \boldsymbol{T}_{P 0}={ }^{{ }^{W}} \boldsymbol{T}_{M 0}{ }^{M 0} \boldsymbol{T}_{60}{ }^{60} \boldsymbol{T}_{C 0}{ }^{C 0} \boldsymbol{T}_{P 0}
$$

The symbol " 0 " attached to the frames in Fig. 1 and to the homogeneous matrices in Eq.(1) expresses the reference relationship of position/orientation. Those frames and matrices are known, because they are set as desired values. On the other hand, in contrast with the desired relationship $A$, the mobile manipulator actually travels to the position illustrated by dotted lines at $B$ in Fig. 1 and experiences traveling errors. This relationship between the mobile manipulator and the landmark is called the actual relationship of position/orientation.

When the relationship of position/orientation between the hand, the camera and the posture of the mounted manipulator is set as ${ }^{M 0} \boldsymbol{T}_{60}{ }^{60} \boldsymbol{T}_{C 0}$, relationship $B$ is expressed by:

$$
{ }^{W} T_{P 0}={ }^{W} T_{M}{ }^{M 0} T_{60}{ }^{60} T_{C 0}{ }^{C} T_{P 0}
$$

In the reference relationship $A$, the posture ${ }^{M 0} T_{B 0}$ for stacking blocks is set on the assumption that the mobile manipulator places at ${ }^{W} T_{M 0}$. Thus, the mobile manipulator at ${ }^{W} T_{M}$ can not stack blocks because of the effects of traveling errors. Thus, it is necessary to determine the posture ${ }^{M} T_{B 0}$ of the mounted manipulator for stacking blocks at ${ }^{W} T_{B 0}$ when the mobile manipulator places at ${ }^{W} T_{M}$. Then, an error of position/orientation of the mobile manipulator is defined by the homogeneous translation matrix $\Delta T_{M}$ shown in Eq.(3).

$$
{ }^{W} T_{M}={ }^{W} T_{M 0} \Delta T_{M}
$$

In addition, an error of position/orientation between ${ }^{C} T_{P 0}$ and ${ }^{C} T_{P 0}$ is defined by the homogeneous translation matrix $\Delta \boldsymbol{T}_{C}$ shown in Eq.(4).

$$
{ }^{C 0} T_{P 0}=\Delta T_{C}{ }^{C} T_{P 0}
$$

Then, a traveling error $\Delta T_{M}$ is obtained from the following equation using Eqs.(1) through (4).

$$
\Delta T_{M}={ }^{M 0} T_{60}{ }^{60} T_{C 0} \Delta T_{C}{ }^{60} T_{C 0}^{-1}{ }^{M 0} T_{60}^{-1}
$$

$\Delta T_{C}$ can be calculated from Eq.(4) by detecting ${ }^{C} T_{P 0}$ from a camera image. Then, $\Delta T_{M}$ is obtained from Eq.(5).

Let a posture of the mounted manipulator be ${ }^{M} \boldsymbol{T}_{60}$ in order to change ${ }^{C} \boldsymbol{T}_{P 0}$ to ${ }^{C 0} \boldsymbol{T}_{P 0}$ in Eqs.(1) through (3), and the following equation is obtained.

$$
{ }^{M} T_{60}=\Delta T_{M}^{-1}{ }^{M 0} T_{60}
$$

${ }^{M} T_{B 0}$ is obtained by Eq.(7) using Eq.(6) and ${ }^{M} \boldsymbol{T}_{B 0}={ }^{M} \boldsymbol{T}_{60}{ }^{60} \boldsymbol{T}_{B 0}$.

$$
{ }^{M} T_{B 0}=\Delta T_{M}^{-1}{ }^{M 0} T_{B 0}
$$

When the mobile manipulator places at ${ }^{W} T_{M}$ and the posture of the mounted manipulator is given by Eq.(6), Eq.(8) is obtained from Eqs.(3) and (7). Therefore, the mobile manipulator can stack blocks at ${ }^{W} T_{B 0}$.

$$
\begin{aligned}
{ }^{W} T_{M}{ }^{M} T_{B 0} & ={ }^{W} T_{M 0} \Delta T_{M} \Delta T_{M}^{-1}{ }^{M 0} T_{B 0} \\
& ={ }^{W} T_{B 0}
\end{aligned}
$$

\subsection{Utilization of Fixation Control}

The camera position in the relationship ${ }^{C 0} \boldsymbol{T}_{P 0}$ is chosen in order to obtain a landmark at the center of a camera image for extending a detecting area of $\Delta T_{C}$. Therefore, when the mobile manipulator places at ${ }^{W} T_{M}$, the camera can not get the landmark at the center of image. Then, ${ }^{C} \boldsymbol{T}_{P 0}$ includes errors caused by a deformation of the lens. In Reference [10], it is shown that a deformation around the edge of a lens is bigger than that of the center. Thus, it is problem that $\Delta T_{C}$, which is calculated by ${ }^{C} T_{P 0}$, includes errors. A fixation control method is utilized in order to remove errors due to the deformation. The position of camera is adapted by the motion of the hand in order to detect a landmark image at the center of lens. Then, this method eliminates the above mentioned errors. Changes in the posture of a mounted manipulator is called fixation motion. The fixation control method is a closed loop and decreases kinematic errors of the mounted manipulator through feedback. Here, the number of the fixation motion is described by $k$. Then, since only 


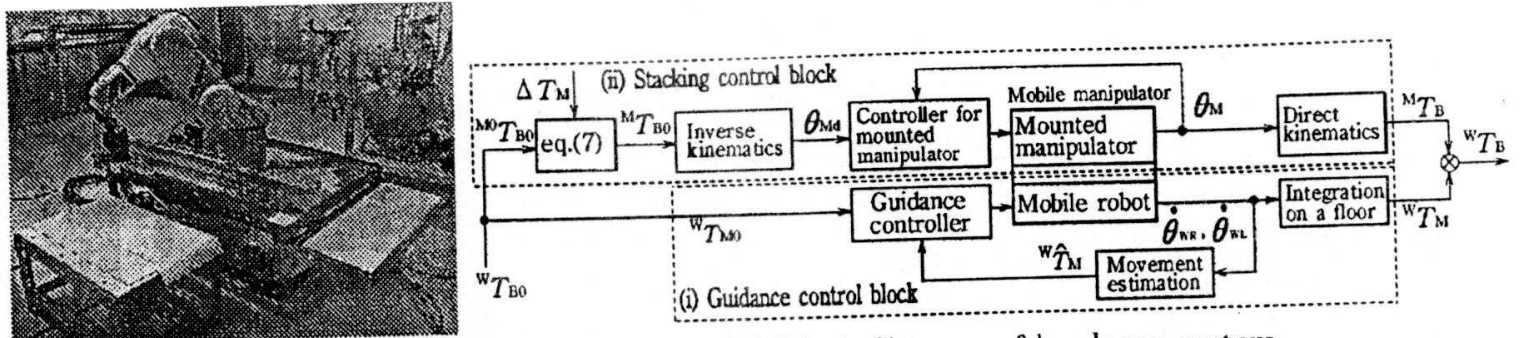

(a) Photo of a mobile manipulator

(b) Block diagram of hardware system

Fig. 2 Our basic operation system

the posture ${ }^{M} \boldsymbol{T}_{60}$ of the mounted manipulator and the relationship ${ }^{C} \boldsymbol{T}_{P_{0}}$ change while fixating, the matrices are described as ${ }^{M} T_{60}^{k}$ and ${ }^{C} T_{P 0}^{k}$ with $k$. In addition, $\Delta \boldsymbol{T}_{C}$, which is derived from ${ }^{C} T_{P 0}^{k}$ using Eq.(4), is also described as $\Delta T_{C}^{k}$ with $k$. Equations (1), (2) and (4) lead to the following equation.

${ }^{W} \boldsymbol{T}_{M 0}{ }^{M 0} \boldsymbol{T}_{60}{ }^{60} \boldsymbol{T}_{C 0} \Delta \boldsymbol{T}_{C}^{k}={ }^{W} \boldsymbol{T}_{M}{ }^{M} T_{60}^{k}{ }^{60} \boldsymbol{T}_{C 0}$

${ }^{M} \bar{T}_{60}$ expresses the final posture of the mounted manipulator after repeated fixation motions in order to make $\Delta T_{C}^{k}$ be unit matrix $I$; i.e., ${ }^{C} T_{P 0}^{k}$ equals ${ }^{C} \boldsymbol{T}_{P 0}$. Then, Eq.(10) is obtained in spite of Eq. (9).

$$
{ }^{W} T_{M 0}{ }^{M 0} T_{60}{ }^{60} T_{C 0}={ }^{W} T_{M}{ }^{M} \tilde{T}_{60}{ }^{60} T_{C 0}
$$

Equations (3) and (10) lead to Eq.(11).

$$
{ }^{M} \tilde{T}_{60}=\Delta T_{M}^{-1{ }^{M 0}} T_{60}
$$

Equation (11) is rewritten as $\Delta T_{M}=$ ${ }^{M}{ }^{0} \boldsymbol{T}_{60}{ }^{M} \tilde{\boldsymbol{T}}_{60}^{-1}$. Then, the traveling error $\Delta T_{M}$ is obtained after removing the detecting error, which was caused by a deformation of the lens, from ${ }^{C} \boldsymbol{T}_{P 0}$ by the image of the landmark in the center of the lens. In addition, since the righthand side of Eq.(11) equals the right-hand side of Eq. (6), thus ${ }^{M} \boldsymbol{T}_{60}={ }^{M} \tilde{\boldsymbol{T}}_{60}$. The posture ${ }^{M} \tilde{\boldsymbol{T}}_{60}$ of the mounted manipulator, which is realized after repeated fixating, is the posture obtained when the traveling error $\Delta T_{M}$ is corrected.

\section{Construction Robot System}

Fig. 2(a) shows a photo of a prototype of our mobile manipulator and Fig. 2(b) shows a block diagram of the constructed system. At first, ${ }^{W} T_{B 0}$, which is an input to the system and is a desired position for stacking blocks, is separated into the desired posture ${ }^{M 0} T_{B 0}$ of the rnounted manipulator and the desired position /orientation ${ }^{W} \boldsymbol{T}_{M 0}$ of the mobile manipulator at the left end of Fig. 2(b). Guidance control is performed in order to navigate the mobile manipulator to a position for stacking blocks in the guidance control block (i), in which ${ }^{W} T_{M 0}$ is input. The control method that was proposed in Reference [7] and the dead-reckoning method in References [4] and [8] are utilized for guidance control.

The movement estimation block for traveling position, which is shown in the lower section of the control block (i), calculates an estimated value of ${ }^{W} \hat{T}_{M}$ of the position/orientation ${ }^{W} T_{M}$ of the mobile manipulator from angular velocities $\dot{\theta}_{W R}$ and $\dot{\theta}_{W L}$ of the right and left driving wheels, respectively. The guidance controller navigates the mobile manipulator using the estimated ${ }^{W} \hat{T}_{M}$. The block "Integration on a floor" shown at the right end of (i) expresses that an integrated traveling result on a road appears as ${ }^{W} T_{M}$.

Here, a locomotion in the real world allows ${ }^{W} \boldsymbol{T}_{M} \neq{ }^{W} \hat{\boldsymbol{T}}_{M}$ and a correct locomotion to ${ }^{W} \boldsymbol{T}_{M 0}$ is difficult [4],[7]:[8]. In the stacking control block (ii), the desired posture ${ }^{M} T_{B 0}$ of a mounted manipulator for stacking blocks is determined from Eq.(7) by using $\Delta T_{M}$, which is determined from Eq.(5) and ${ }^{M 0} T_{B 0}$. Then, the desired angular vector $\theta_{M d}$ of the mounted manipulator for realizing ${ }^{M} T_{B 0}$ is calculated and the controller for the mounted manipulator realizes the posture of the manipulator by using $\theta_{M d l}$. The actual posture ${ }^{M} T_{B}$ of the manipulator is given by using the angular vector $\theta_{M}$ of the manipulator, which is a result of motion, through a direct translation of kinematics.

The right end of Fig. 2(b) shows that the position of a stack of blocks with reference to $\Sigma_{W}$ becomes ${ }^{W} T_{B}$ by using ${ }^{W} T_{M}$ and ${ }^{M} T_{B}$.

\section{Influences by A Mobile Robot}

Changes in yaw angle of a mobile robot are produced by dynarnical interactions between the mounted manipulator and the mobile robot during operation. In addition, changes in pitch angle of a mobile robot occur with movements of a suspension mechanism, which is attached to the bottom of the mobile robot. The movement of the suspension is generated by changes in the position of the center of gravity with changes in posture of the mounted manipulator during operation. Those changes in yaw and pitch angles affect the position of the hand. This problem is one of identical problems of a mobile manipulator having a moving mechanism with suspension. Reference [5], in which influences on the position of the hand caused by suspension are discussed, has been publishcd. However, the research did not report positioning errors of objects when the mobile manipulator operates to an external world.

First, the posture of the mobile manipulator after fixation motion is completed at loading and 


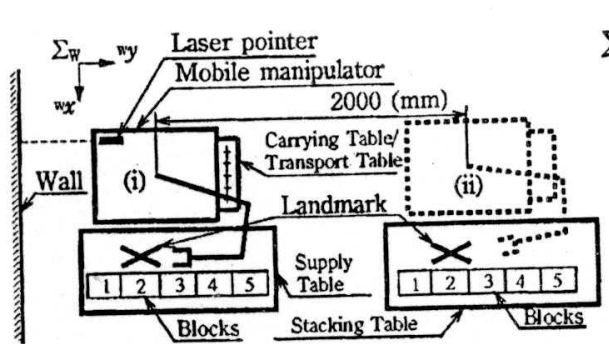

(a) Aerial view of experiment equipment

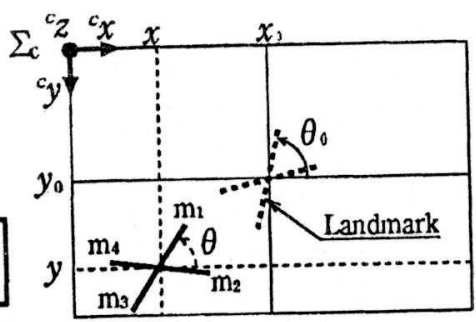

(b) Landmark Image

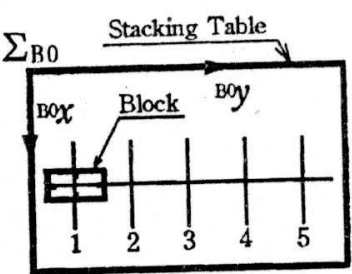

(c) Desired positions of blocks

Fig. 3 Experimental equipment

unloading positions, which are (i) and (ii), is set as a reference posture. Then, changes in yaw angle of $\boldsymbol{\Sigma}_{M}$ around the ${ }^{M} z$ axis are expressed by $\Delta \psi$ and changes in pitch angle of $\Sigma_{M}$ around the ${ }^{M} x$ axis are described by $\Delta \phi$.

Changes in orientation of the frame $\boldsymbol{\Sigma}_{M}$ caused by $\Delta \psi$ are expressed by a homogeneous matrix $\Delta \boldsymbol{\Psi}$ and changes produced by $\Delta \phi$ are expressed by $\Delta \Phi$. The change in the position of the hand ${ }^{M} T_{B}$ generated by $\Delta \Psi$ and $\Delta \Phi$ is cxpressed by ${ }^{M} \boldsymbol{T}_{B}$. Then, ${ }^{{ }^{M} T_{B}}$ is given by:

$$
{ }^{M} T_{B}=\Delta \Psi \Delta \Phi^{M} T_{B 0}
$$

\section{Experiments on Stacking Blocks}

\subsection{Experimental Procedure}

The positioning accuracy of the stacked blocks is evaluated using the proposed construction system. In the present experiments, a situation in which a partition wall in a condominium is constructed by blocks is considered. It is assumed that the plane in which the manipulator travels and stacks the blocks is flat. The blocks used in the experiment were made of wood and were $25 \mathrm{~mm}$ in length, $80 \mathrm{~mm}$ in width and $40 \mathrm{~mm}$ in height. Fig. 3(a) shows an aerial view of the experimental equipment. In Fig. 3(a), three tables, which are located at points (i) and (ii) and attached to the mobile manipulator, are for the supply, the stack and the transportation of blocks, respectively. Landmarks are attached to both the supply and stacking tables. The number of blocks on the supply and stacking tables expresses the order of carrying and stacking blocks. Desired positions for stacking blocks are set as $90 \mathrm{~mm}$ far from the previous blocks along ${ }^{W} y$ with reference to the 1st block. Changes in yaw and pitch angles mentioned in Section 4 are calculated from an irradiated point on the wall by the laser pointer shown in Fig. 3(a) and the distance between the laser pointer and the wall. The experimental procedure of the basic stacking blocks is as follows.

(1) $\Delta T_{M}$ is recognized as an initial error of position/orientation of the mobile manipulator by performing fixation motion at (i) in Fig. 3(a). Then, five blocks are placed on the transport table from the supply table by the mounted manipulator's hand.

(2) The mobile manipulator travels to point (ii) using the dead-reckoning method.

(3) The traveling error $\Delta T_{M}$ is recognized by the fixation motion at the point (ii).
(4) The desired stacking position ${ }^{M} T_{B 0}$ is calculated from Eq.(7).

(5) The mounted manipulator moves in order to realize the posture ${ }^{M} T_{B 0}$ and places the five blocks on the stacking table at (ii).

\subsection{Recognition of Traveling Errors}

The method for determining $\Delta T_{C}$ is as follows. When the hand is faced to the perpendicular below at $B$ in Fig. 1, the image illustrated by the solid lines in Fig. 3(b) is obtained. The landmark drawn by dotted lines in the figure is the reference image obtained in $A$. It is necessary to sense the position in the ${ }^{C} x$ and ${ }^{C} y$ directions and the rotational angle around ${ }^{C} z$ axis at $\Sigma_{W}$ in order to recognize a traveling error. Thus, any shape of landmark can be employed, if the above mentioned position and orientation can be sensed. The point of intersection of the diagonal lines, which are obtained by determining the end points $m_{1}$ through $m_{4}$, is calculated in order to recognize the center position of the landmark. In addition, the orientation of the landmark is calculated by angle $\theta$ with reference to ${ }^{C} x$ axis. ${ }^{C} T_{P 0}$ is obtained from the calculated position and orientation. $\Delta T_{C}$ is determined from ${ }^{C} \boldsymbol{T}_{P 0}$ because ${ }^{C 0} T_{P 0}$ is known. Then, the fixation motion, which is described in Subsection 2.2 , is performed. However, it is difficult to perform the fixation control repeatedly till $\Delta T_{C}^{k}$ fits the unit matrix $I$ completely, because of the absolute positioning accuracy of the mounted manipulator. Therefore, the fixation motion is terminated when the following conditions are satisfied.

$$
\left|x_{0}-x\right| \leq \epsilon_{1},\left|y_{0}-y\right| \leq \epsilon_{1},\left|\theta_{0}-\theta\right| \leq \epsilon_{2}
$$

where $|\cdot|$ is an absolute value and $\epsilon_{1}$ and $\epsilon_{2}$ are constant values in order to define the conditions to terminate the motion.

\subsection{Evaluation Method of Stacking Errors}

An enlargement of the stacking table is shown in Fig. 3(c). The numbers 1 through 5 express the order in which the blocks will be stacked. Cross lines are painted on the table in order to define the positions at which the blocks will be stacked. Lines are painted at the midpoint of each side of the bottom of the blocks. When the lines on the blocks coincide with the lines on the table, the stacking error is zero. The $x$ and $y$ coordinates of 

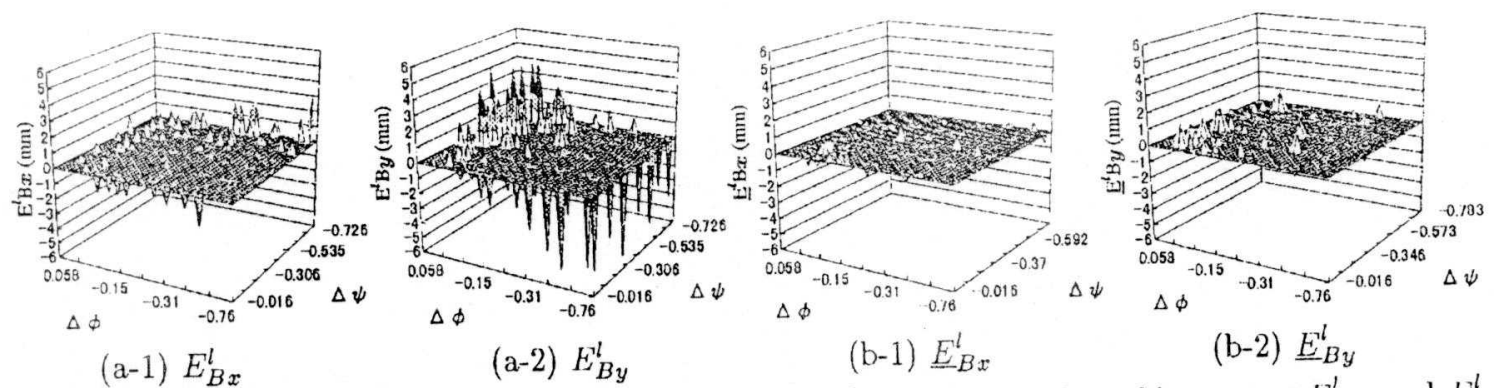

Fig. 4 Relationships among stacking errors $E_{M C x}^{l}$ and $E_{M C y}^{l}$, processed stacking errors $\underline{E}_{B x}^{l}$ and $\underline{E}_{B y}^{l}$ aftcr eliminating the effects of changes in yaw and pitch angle, $\Delta \psi$ and $\Delta \phi$

${ }^{M} T_{B}$ are expressed by ${ }^{M} x_{B}$ and ${ }^{M} y_{B}$. In addition, the ${ }^{W} x$ and ${ }^{W} y$ coordinates of ${ }^{W} T_{B 0}$ and ${ }^{W} T_{B}$ are expressed by $w_{x_{B 0}}, W_{y_{B 0}}, w_{x_{B}}$ and ${ }^{W_{y_{B}}}$, respectively. Errors along the ${ }^{B 0} x$ and ${ }^{B 0} y$ axes of $\Sigma_{B O}$ are expressed from $E_{B x}$ and $E_{B y}$. Then, $E_{B x}$ and $E_{B y}$ are obtained by Eq. (14) as the difference between ${ }^{W} x_{B 0}$ and ${ }^{W} x_{B}$ or $w_{y_{B 0}}$ and $w_{y_{B}}$. Here, the symbol $l(l=1 \sim 5)$ is the number of the block. The errors defined by Eq.(11) are measured on a scale with $0.5 \mathrm{~mm}$ steps.

$$
E_{B \beta}^{l}={ }^{W} \beta_{B 0}^{l}-{ }^{W} \beta_{B}^{l}(\beta=x, y)
$$

\subsection{Errors of Yaw and Pitch Angles}

In this subsection, the effects on stacking accuracy of change in hand position due to changes in yaw and pitch angles are evaluated. In the present experiments, the mobile manipulator unloaded the blocks from the transport table and stacked on the stacking table. The task of stacking five blocks was set as one trial, and 20 trails were performed; i.e., 100 blocks were stacked. The absolute positioning accuracy of the hand is within $0.5 \mathrm{~mm}$, as estimated from preliminary experiments. The conditions $\epsilon_{1}$ and the $\epsilon_{2}$ were set as $\epsilon_{1}=0.5 \mathrm{~mm}$ and $\epsilon_{2}=0.2 \mathrm{rad}$ by considering convergence time. Thus, an error occurs within $0.5 \mathrm{~mm}$ during a carrying operation and a stacking operation, respectively, and the condition $\epsilon_{1}$ allows an error of less than $0.5 \mathrm{~mm}$. Therefore, a positioning error must not exceed $1.5 \mathrm{~mm}$ in total. Figs. 4 (a-1) and (a-2) show errors $E_{B \beta}^{l}(\beta=x, y)$ in the position of stacked blocks with measured $\Delta \psi$ and $\Delta \phi$. Points where data does not exist or where the error is zero are plotted as zero on the error axis. In Fig. 4(a-1), no correlative relationships between the error and the changes in yaw and pitch angles can be seen. The reason is that a position of the block in the case of unloading is corrected to a position of the hand, because the opening/closing power of the hand is larger than the friction force between the block and the surface of the table. Fig. 4(a-2) shows that the errors increased in a positive direction as $\Delta \psi$ and $\Delta \phi$ increased in the negative direction and to the positive direction, respectively.

It is clear that changes in yaw and pitch angles increased the errors. Thus, it is necessary to eliminate the effects of changes in yaw and pitch angles. It was thought that these effects could be eliminated by subtracting the changes in hand position, which are calculated by Eq.(12), from stacking errors. After eliminating effects on hand position, errors $E_{B x}^{l}$ and $E_{B y}^{l}$ are expressed by $\underline{E}_{B x}^{l}$ and $\underline{E}_{B y}^{l}$ and are shown in Figs. $4(\mathrm{~b}-1)$ and (b-2). The absolute values of the processed errors shown in Figs. 4(a-1) and (a-2) are within $1.5 \mathrm{~mm}$. Thus, the increase in the stacking errors shown in Figs. $4(a-1)$ and $(a-2)$ is caused by changes in yaw and pitch angles of the mobile robot.

\subsection{Results of Basic-Operation Experiment}

Next, the stacking accuracy of the basic operation shown in (1) through (5) of Subsection 5.1 is evaluated. Figs. $5(\mathrm{a}-1)$ and $(\mathrm{a}-2)$ show stacking accuracy $E_{B x}^{i}$ and $E_{B y}^{l}$. In these figures, the $x$ axis shows the trial number, the $y$ axis shows the number of blocks, and the $z$ axis shows the errors of the stacked blocks $E_{B x}^{l}$ and $E_{B y}^{l}$, respectively.

Fig. 5(a-1) shows that the error $E_{B x}^{l}$ increased with the increase in the number of blocks. However, this tendency can not be seen in error $E_{B y}^{l}$ in (a-2). The reason for this is that the error along the ${ }^{B 0} x$ axis is corrected to the position of the hand, through the influence of the opening/closing power of the hand. Then, the effects of the changes in yaw and pitch angles during the carrying operation do not appear. Thus, the error depends on only the changes that occur during the stacking of the blocks. In contrast, the error along the ${ }^{B 0} y$ axis depends on both the effects of the changes during the loading and stacking of the blocks. Thus, the above mentioned tendency does not appear in the error $E_{B y}^{l}$.

Fig. $\bar{\jmath}(\mathrm{b}-1)$ shows the processed error $\underline{E}_{B x}^{l}$ and $\underline{E}_{B y}^{l}$ of $(\mathrm{b}-2)$ after eliminating the effects of changes in yaw and pitch angles. Figs. 5(b-1) and (b-2) indicate that the absolute values of the processed errors are within $3 \mathrm{~mm}$. Therefore, as in the previous subsection, the increases in the errors were generated by the effects in the yaw and pitch angles. In addition, except for this influence, the error produced by the absolute positioning accuracy of the mounted manipulator's hand and the fixation motion. This means that the proposed construction robot system can detect traveling errors and can stack blocks at the desired positions against traveling errors in the error range of the absolute positioning accuracy of hand and fixation 


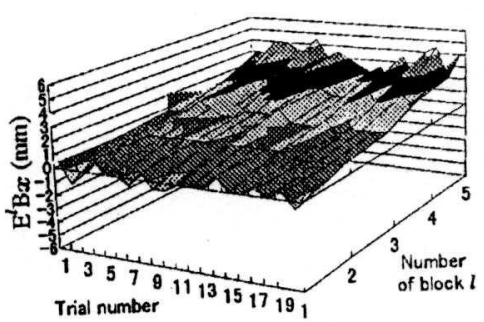

(a-1) $E_{B x}^{l}$

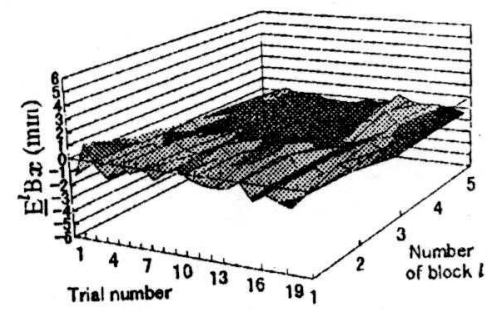

(b-1) $\underline{E}_{B x}^{l}$
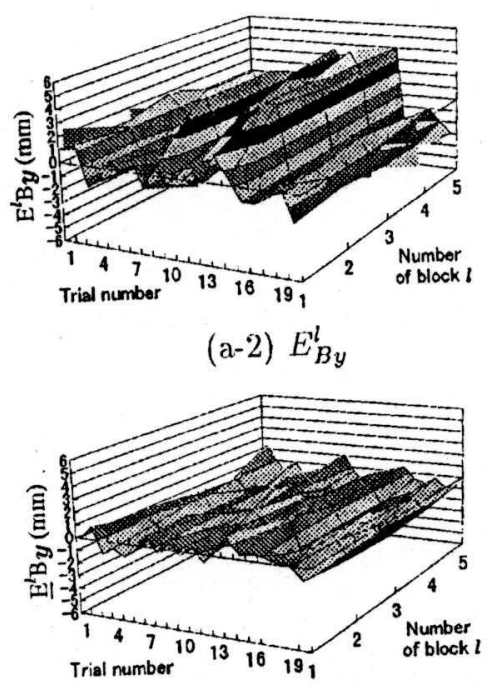

(b-2) $\underline{E}_{B y}^{l}$

Fig. 5 Relationships among errors $E_{B x}^{l}, E_{B y}^{l}$, processed errors $\underline{E}_{B x}^{l}: \underline{E}_{B y}^{l}$ after eliminateing the effects of changes in yaw and pitch angles, trial number and number of blocks

control when changes in yaw and pitch angles do not occur.

\section{Conclusions}

In the present paper,

(1) We proposed a basic construction robot system that uses a mobile manipulator having a compensation system, which includes a handeye camera, landmarks and a fixation motion, for piling up errors caused by traveling errors

(2) Experiments on the basic opcration of stacking blocks, in which the blocks are carried, transported and then stacked on tables, were performed with a prototype of our experimental equipment. The magnitude of errors of stacked blocks has also been evaluated.

(3) It has been shown that changes in yaw and pitch angles affect stacking accuracy. Thus, the relationships between these changes and stacking errors were discussed.

(4) From the experimental results, it was shown that the proposed method is able to compensate for traveling errors, which are the dominant parameters in generating stacking errors. Thus, when the effects of changes in yaw and pitch angles are eliminated, the remaining stacking errors are dependent on the absolute positioning accuracy of the mounted manipulator. Therefore, therc is a possibility that the proposed system can be implemented on the construction industry.

\section{REFERENCES}

[1] T.Fukuda, Y.Fujisawa, K.Kosuge, F.Arai, E.Muro, H.Hoshino, T.Miyazaki, K.Ohtsubo, K.Uehara. "Manipulator for Man-Robot Cooperation Work(3rd Report, Control Algorithm of Center of Gravity for Manipulator/Vehicles System)" Transactions of the Japan Society of Mechanical Engineers, 58-551 C, p.2152-2158, (1992), (in Japanese)
[2] Y.Yamamoto, X.Yun. "Modeling and Compensation of the Dynamic Interaction of a Mobile Manipulator" Proc. of IEEE Int. Conf. on Robotics and Automation, p.2187-2192, 1994.

[3] M.Kurisu, T.Yoshikawa. "Trajectory Planning and Dynamic Control of a Mobile Manipulator" Transactions of the Japan Society of Mechanical Engineers, 62-596 C, p.1488, (1996), (in Japanese).

4] M.Minami, N.Fujiwara, H.Tsuge. "Position - Velocity Control of Autonomous Mobile Manipulator with Camera Feedback" Journal of Robotics Society of Japan, Vol.11, No.2, p.263-271, (1993), (in Japanese).

[5] N.M.Hootsmans, S.Dubowsky. "Large Motion Control of Mobile Manipulators Including Vehicle Suspension Characteristics" Proc. of the 1991 IEEE Int. conf. on Robotics and Automation, p.2336-2341, 1991.

[6] Y.Nakamura. "Nonholonomic Robot Systems(Part 2:Motion Planning under Kinematic Nonholonomic Constaints)" Journal of Robotics Society of Japan, Vol.11, No.5, p.655-662, (1993), (in Japarnese).

[7] Y.Kanayama, Y.Kimura, F.Miyazaki, T.Noguchi. "A stable tracking control method for an alltonomous mobile Robot" Proc. of Int. Conf. on Rob. and Autom., p.384-349, 1990.

[8] T.Komori, S.Tachi, K.Tanie. "A Method for Autonomous Locomotion of Mobile Robots" Journal of Robotics Society of Japan, Vol.2, No.3, p.222231, (1984), (in Japanese).

[9] T.Yagi, N.Asano, S.Makita, Y.Uchikawa. "Active Vision System Inspired by Biological Fixation Mechanism" Transactions of the Japan Society of Mechanical Engineers, 61-591 C, p.4402-4409, (1995): (in Japanese).

[10] M.Kume, D.Takaoka, M.Yamada, K.Tsukamoto. T.Kanade. "A Calibration Method Based on the Distortion Correction by Neural Networks" Transactions of the Japan Society of Mechanical Engineers, 61-591 C, p.4436-4442, (1995). (in Japanese). 